\author{
Military Technical College \\ Kobry El-Kobbah, \\ Cairo, Egypt.
}

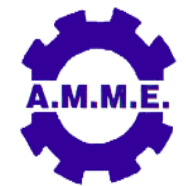

$18^{\text {th }}$ International Conference on Applied Mechanics and Mechanical Engineering.

\title{
EXPERIMENTAL AND NUMERICAL INVESTIGATION OF DRAG FORCE REDUCTION OVER UNDERWATER VEHICLES
}

\author{
T. A. Ghonim* and M. S. Farag*
}

\begin{abstract}
This paper concerns with investigating experimentally and numerically two different methods of drag force reduction over underwater vehicles. The first method is the viscous friction drag reduction by air injection in the near wall layer and the second one is the pressure drag reduction by changing the vehicle nose profile. An experimental test rig was designed and manufactured with all required measurements of drag force and water velocity. Additionally, the experimental work was conducted on six underwater vehicle (torpedo) models with different nose profiles and lengths at variable water velocities. Computational Fluid Dynamic (CFD) simulation was performed using ANSYS. Furthermore, the computations were extended to depict the velocity and pressure contours around two models having different body nose profiles at water velocity of $50 \mathrm{~m} / \mathrm{s}$. The total drag forces over eight models were predicted and compared. The numerical results showed good agreement with the experimental ones. The experimental results showed that, for speed range of $1-2.5 \mathrm{~m} / \mathrm{s}$ the stubbed nose profile had the highest drag. On the other hand, the stepped conical nose profile had the lowest drag force. Moreover, the experimental results of air injection showed a decrease in drag force in the velocity range of $1.7-2.5 \mathrm{~m} / \mathrm{s}$ but an increase in the drag for velocity range of $1-1.7 \mathrm{~m} / \mathrm{s}$ was remarked. Finally, there was a significant decrease in drag force of elliptical nose model compared to other models over the velocity range of (0 to $50 \mathrm{~m} / \mathrm{s}$ ).
\end{abstract}

\section{KEY WORDS}

Drag force reduction, CFD, air injection, nose profile, and viscous friction drag.

* Lecturer, Dept. of Mech. Power Engineering, Faculty of Engineering, Menoufia University, Egypt. 


\section{INTRODUCTION}

Drag force is defined as the total resistance force which is applied on a body. This resistance force can be decomposed into two different components: viscous friction resistance, and pressure resistance. Drag reduction is a one of important techniques to achieve fuel consumption reduction on marine vehicles. Obviously, any reduction in the drag experienced by say, a ship, means less fuel consumption. This is why over the years different techniques have been developed to reduce the drag in internal and external flows [1-2]. Mohanarangam et al. [3] studied the skin friction reduction by introduction of micro-bubbles into turbulent boundary layer. Their study discussed the various physical phenomenon's causing the drag reduction along the boundary layer, firstly it was observed that there is drop in the mean streamwise water velocities with a subsequent increase in the drag along varying gas injection rates. Secondly, the presence of the micro-bubbles caused turbulence attenuation for some distance along the boundary layer and later an augmentation was felt due to the shedding of the vortices behind the bubbles. Drag reduction by polymer additives in a turbulent channel flow were studied by Minet al. [4]. Turbulent drag reduction by polymer additives in a channel is investigated using direct numerical simulation. Watanabe [5] investigated the drag reduction by fluid slip. Drag reduction phenomenon of a highly water-repellent wall was presented referring the experimental results for a pipe flow and a flow around a sphere. It was experimentally clarified that the laminar drag reduction was caused by the fluid slip resulting from slip velocity generated by a gas-liquid interface at the wall with fractal structure. Turbulent drag reductions with polymers in rotating disk flow were introduced by Hong et al. [6]. The frictional drag in turbulent flow can be drastically reduced by the addition of minute amounts of suitable linear flexible high-molecular-weight polymers, and the various physical characteristics of the polymers used are known to be closely related to the drag reduction efficiency. This feature article briefly reviews polymer additives and factors in the system affecting turbulent drag reduction in external flow, more specifically in a rotating disk flow. Tianet al. [7] studied an optimization method for the design of the layout of an autonomous underwater vehicles (AUV) fleet to minimize the drag force. The layout of the AUV fleet was defined by two nondimensional parameters. Three-dimensional computational fluid dynamics (CFD) simulations were performed on the fleets with different layout parameters and detailed information on the hydrodynamic forces and low structures around the AUVs. Drag reduction for high-speed underwater vehicles was studied by Nesteruk [8]. The underwater hulls drag reduction was investigated analytically and numerically with the use of the axisymmetric flow of the ideal and the viscous fluid approaches. Different effectiveness criteria, such as: the volumetric drag coefficient, the drag coefficients, based on the maximum body cross-section area and the squared hull length, and the ranges of the inertial motion. S. Jafargholinejad [9] presented an investigation of hydrophobic polymer coating effect on autonomous underwater vehicles skin friction reduction. Applying hydrophobic coating on control surface and measuring drag force exerted on a model in a water channel, leads to achieving an optimum mechanical performance and fuel consumption reducing of an autonomous underwater vehicle system.

The main motivation behind the present work is to fulfill the following:

1. Develop and validate a 3Dmathematical model to predict the viscous, pressure, and total drag forces over underwater vehicles. 
2. Design and fabrication of an experimental setup for total drag force measurements to validate the proposed mathematical model.

3. Studying experimentally the feasibility of viscous friction drag force reduction by air injection on the surface of an underwater vehicle.

4. Investigating numerically the effect of the underwater vehicle head (nose) profile on both viscous friction and pressure drag forces.

\section{MATHEMATICAL MODEL}

This section includes the main equations which describe the drag force prediction over underwater vehicles. In the present study, the theoretical model is formulated based on the following assumptions: steady, single phase, turbulent and viscous incompressible flow.

\section{Equation of Continuity}

$$
\frac{\partial}{\partial x_{i}}\left(u_{i}\right)=0
$$

\section{Momentum Equation}

$$
\frac{\partial}{\partial x_{j}}\left(\rho u_{i} u_{j}\right)=-\frac{\partial p}{\partial x_{i}}+\frac{\partial}{\partial x_{j}}\left[\mu\left(\frac{\partial u_{i}}{\partial x_{j}}+\frac{\partial u_{j}}{\partial x_{i}}-\frac{2}{3} \delta_{i j} \frac{\partial u_{l}}{\partial x_{l}}\right)\right]+\frac{\partial}{\partial x_{j}}\left(-\rho \overline{u_{i}^{\prime} u_{j}^{\prime}}\right)
$$

where, $u_{i}$ denotes the mean velocities and $u_{i}^{\prime}$ and $u_{j}^{\prime}$ are the turbulent fluctuations, $\rho_{\text {is }}$ the density and $\mathrm{p}$ is the pressure. The momentum equations contain additional terms, known as Reynolds stresses that represent the effects of turbulence. These Reynolds stresses, $-\rho \overline{u_{i}^{\prime} u_{j}^{\prime}} \quad$ must be modeled in order to close governing system of equations. That can be done through the appropriate choice of the turbulence model.

\section{Turbulence Modeling}

In industrial CFD applications, RANS modeling remains one of the main approaches when dealing with turbulent flows. The Reynolds-averaged approach to turbulence modeling requires appropriate modeling of the Reynolds stresses in Equation 2. The common method to relate the Reynolds stresses to the mean velocity gradients is based on Boussinesq hypothesis [10]:

$$
-\rho u_{i}^{\prime} u_{j}^{\prime}=\mu_{t}\left(\frac{\partial u_{i}}{\partial x_{j}}+\frac{\partial u_{j}}{\partial x_{i}}\right)-\frac{2}{3}\left(\rho k+\mu_{t} \frac{\partial u_{k}}{\partial x_{k}}\right) \delta_{i j}
$$

where, $\delta_{i j}$ is the Kronecker delta function ( $\delta_{i j}=1$ if $i=j$ and $\delta_{i j}=0$ if $i \neq j$ ), $k$ is the turbulent kinetic energy. One of the most challenging problem of turbulent flow is the estimation of the turbulent viscosity, which, unlike the dynamic viscosity, not a flow property. 
For the calculation of the turbulent viscosity, the extended $k-\omega$ turbulence model, where The turbulence kinetic energy, $k$, and the specific dissipation rate, $\omega$, are obtained from the following transport equations [11]:

$$
\frac{\partial}{\partial x_{j}}\left(\rho k u_{i}\right)=\frac{\partial}{\partial x_{j}}\left(\Gamma_{k} \frac{\partial k}{\partial x_{i}}\right)+G_{k}-Y_{k}+S_{k}
$$

and

$$
\frac{\partial}{\partial x_{j}}\left(\rho \omega u_{i}\right)=\frac{\partial}{\partial x_{j}}\left(\Gamma_{\omega} \frac{\partial k}{\partial x_{i}}\right)+G_{\omega}-Y_{\omega}+S_{\omega}
$$

In these equations, $G_{k}$ represents the generation of turbulence kinetic energy due to mean velocity gradients. $G_{\omega}$ represents the generation of $\omega . \Gamma_{k}$ and $\Gamma_{\omega}$ represent the effective diffusivity of $\mathrm{k}$ and !, respectively. $Y_{K}$ and $Y_{\omega}$ represent the dissipation of $k$ and $\omega$ due to turbulence. All of the above terms are calculated as described below. $S_{k}$ and $S_{\omega}$ are user defined source terms [12]. The effective diffusivities for the $k-\omega$ model are given by:

$$
\begin{gathered}
\Gamma_{k}=\mu+\frac{\mu_{t}}{\sigma_{k}} \\
\Gamma_{\omega}=\mu+\frac{\mu_{t}}{\sigma_{\omega}}
\end{gathered}
$$

where $\sigma_{k}$ and $\sigma_{\omega}$ are the turbulent Prandtl numbers for $k$ and $\omega$, respectively. The turbulent viscosity, $\mu_{t}$, is computed by combining $k$ and $\omega$ as follows [12]:

$$
\mu_{t}=\alpha^{*} \frac{\rho k}{\omega}
$$

The production of specific dissipation rate is mathematically defined as [12]:

$$
G_{\omega}=\alpha \frac{\omega}{k} G_{k}
$$

The computational solution domain is shown in Fig. 1. A grid size ofabout 620000 cells was used after a grid independent study was carried out. Finite volume method was used with pressure-based solver. A simple scheme for pressure-velocity coupling and a second order upwind for discretization of governing equations were used.The convergence criteria of the residuals were lower than $10^{-6}$.

\section{EXPERIMENTAL SETUP AND MEASUREMENT PROCEDURES}

The experimental test rig, which was designed and manufactured to perform the measurements of the present work, is illustrated schematically and photographically 
in Fig.2. Water is circulated in a closed loop cycle using a water pump. The circulating water channel dimensions are $6 \mathrm{~m}$ long and $0.7 \mathrm{~m}$ wide. A Pitot-static tube is used to measure the water velocity along the centerline of the experimental model under study. Moreover, the water velocity is changed by a flow control valve fitted on the discharge side of the pump. The drag force on the experimental model under study is measured by a sensitive digital scale which is wired indirectly to the experimental model via a sliding carriage. The experimental model is suspended beneath a sliding carriage mechanism in order to permit free movement of the experimental model due to drag. A photograph of the sliding carriage mechanism, suspension rods and one of the experimental models is shown in Fig. 3.The geometric dimensions of the investigated models are shown in Fig. 3. However, only the first four models in Fig. 4 are experimentally tested. The fifth experimental model of Ref. [9] used in the CFD model validation is shown in Fig. 5. In addition, Fig. 6 shows the sixth experimental model which is tested with air injection through 8 holes of $2 \mathrm{~mm}$ diameter. Air is injected axially in the same direction of the water flow via an air compressor through an opening which is internally connected to the 8 holes illustrated on the model. A photograph of the six experimental models is depicted in Fig. 7.

\section{RESULTS AND DISCUSSION}

The proposed CFD model is validated against both present experimental and experimental data provided by Ref [9] for model (9). It is apparently illustrated that the present CFD model is capable of predicting the total drag force well as depicted in Fig. 8. Figure 9 illustrates experimental drag measurements at low velocity range (1 - $2.5 \mathrm{~m} / \mathrm{s}$ ) of models (1 to 4) which are shown in Fig. 4-b.It is seen that model (3) with the stepped conical nose profile has the lowest total drag force. But, model (4) with the stubbed nose profile has the highest total drag force. Figure 10 shows a comparison of measured total drag force of model (10) with and without air bubbles injection. It is clear that air injection had a negative effect on the total drag force up to a velocity of $1.7 \mathrm{~m} / \mathrm{s}$. However, air injection decreased the total drag force for the velocity values beyond $1.7 \mathrm{~m} / \mathrm{s}$. The effect of eight different nose profiles of the underwater vehicle on pressure drag, viscous friction drag, and total drag forces is investigated numerically and respectively in Figs. 11, 12, and 13. The conical nose profile showed the highest pressure drag while the elliptical nose profile exhibited the lowest drag force. On the other hand, the hemispherical nose profile indicated the highest viscous friction drag but the conical nose profile had the lowest drag. Furthermore, the nose profile had smaller effect on the viscous friction drag than on the pressure drag where the elliptical nose profile shows the lowest total drag force and the conical and stubbed nose have the highest total drag force. Figures 14 and 15 depict the velocity and pressure contours around the stubbed and elliptical nose profile models respectively at water velocity of $50 \mathrm{~m} / \mathrm{s}$. The highest pressure region on the front of the nose is seen to be larger in the stubbed nose than the elliptical one.

\section{CONCLUSION}

The present paper presents a CFD model for predicting the pressure, viscous friction, and total drag forces over underwater vehicles. The proposed CFD model is 
validated against both present and available previously published experiments. The experimental results showed that, for speed range of $1-2.5 \mathrm{~m} / \mathrm{s}$ the stubbed nose profile had the highest total drag force. On the other hand, the stepped conical nose profile had the lowest total drag force. Additionally, the nose profile had very small effect on the viscous drag force predictions in small velocity ranges. However, the nose profile affected greatly the pressure drag force predictions in the whole velocity ranges.

Moreover, the experimental results of air injection showed a decrease in drag force in the velocity range of $1.7-2.5 \mathrm{~m} / \mathrm{s}$ but an increase in the drag for velocity range of 1 $1.7 \mathrm{~m} / \mathrm{s}$ is seen. However, more study on drag reduction by air injection is required to investigate the effect different parameters such as air flow rate, number of air holes, holes diameter and air bubbles size, which is an objective of a future work. The super-cavitation nose profiles 1 and 2 need more numerical investigation at speeds higher than $50 \mathrm{~m} / \mathrm{s}$ after changing the single-phase model to two-phase model. Finally, there was a significant decrease in the total drag force of the elliptical nose profile compared to other models over the velocity range of $(0$ to $50 \mathrm{~m} / \mathrm{s}$ ).

\section{REFERENCES}

[1] F. H. Bark, E. J. Hinch and M. T. Landahl, " Drag reduction in turbulent flow due to additives: a report on Euromech 52", J. Fluid mech. Vol. 68, part 1, PP. 129-138, (1975).

[2] S. Jafargholinejad and K. Sadeghy, "Experimental Investigation on Drag reduction performance of two kind of polymeric coatings with rotating disk apparatus", $18^{\text {th }}$ Annual International Conference on Mechanical EngineeringISME2010, 11-13 May, Sharif University of Technology, Tehran, Iran, (2010).

[3] K. Mohanarangam, C.P. Cheung, J. Y. Tu and L.Chen, "Skin friction Reduction by Introduction of Micro-bubbles into Turbulent Boundary Layer", 16th Australasian Fluid Mechanics Conference, Crown Plaza, Gold Coast, Australia 2-7 December (2007)

[4] Taegee Min, Jung YulYoo, Haecheon Choi and Daniel D. Joseph, "Drag reduction by polymer additives in a turbulent channel flow", J. Fluid Mech. Vol. 486, PP. 213-238, (2003).

[5] K. Watanabe "Drag Reduction by Fluid Slip", American Journal of Science and Technology , Vol. 2. PP. 106-110, (2015).

[6] Cheng Hai Hong , Chun Hag Jang and Hyoung Jin Choi "Turbulent Drag Reduction with Polymers in Rotating Disk Flow", Polymers, Vol. 7, PP. 12791298, (2015).

[7] W. Tian, Z. Mao, F. Zhao and Z. Zhao, "Layout Optimization of Two Autonomous Underwater Vehicles for Drag Reduction with a Combined CFD and Neural Network Method", Hindawi, Complexity, Article ID 5769794, (2017).

[8] I. Nesteruk, "Drag Reduction For High-Speed Underwater Vehicles", the $7^{\text {th }}$ International Symposium on Cavitation CAV2009 - Paper No. 86 August 1722, Ann Arbor, Michigan, USA, (2009).

[9] S. Jafargholinejad, "Investigation of Hydrophobic Polymer Coating Effect on Autonomous Underwater Vehicles Skin Friction Reduction", the $11^{\text {th }}$ International Conference on Coasts, Ports and Marine Structures (ICOPMAS 2014) Tehran, Iran, 24-26 Nov. (2014). 
[10] Hinze, J. O., Turbulence, McGraw-Hill Publishing Co., New York, (1975).

[11] Wilcox D. C., "Turbulence Modeling for CFD", DCW Industries, Inc., La Canada, California, (1998).

[12] Fluent, User's Guide Fluent 6.3.26, Fluent Incorporated, Lebanon, NH, (2006). 


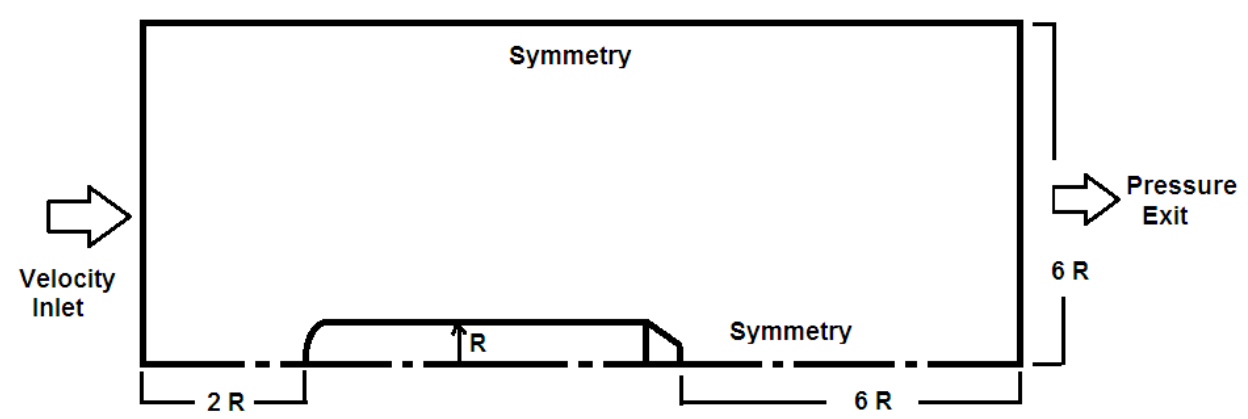

Fig. 1. Boundary conditions and the computational domain.

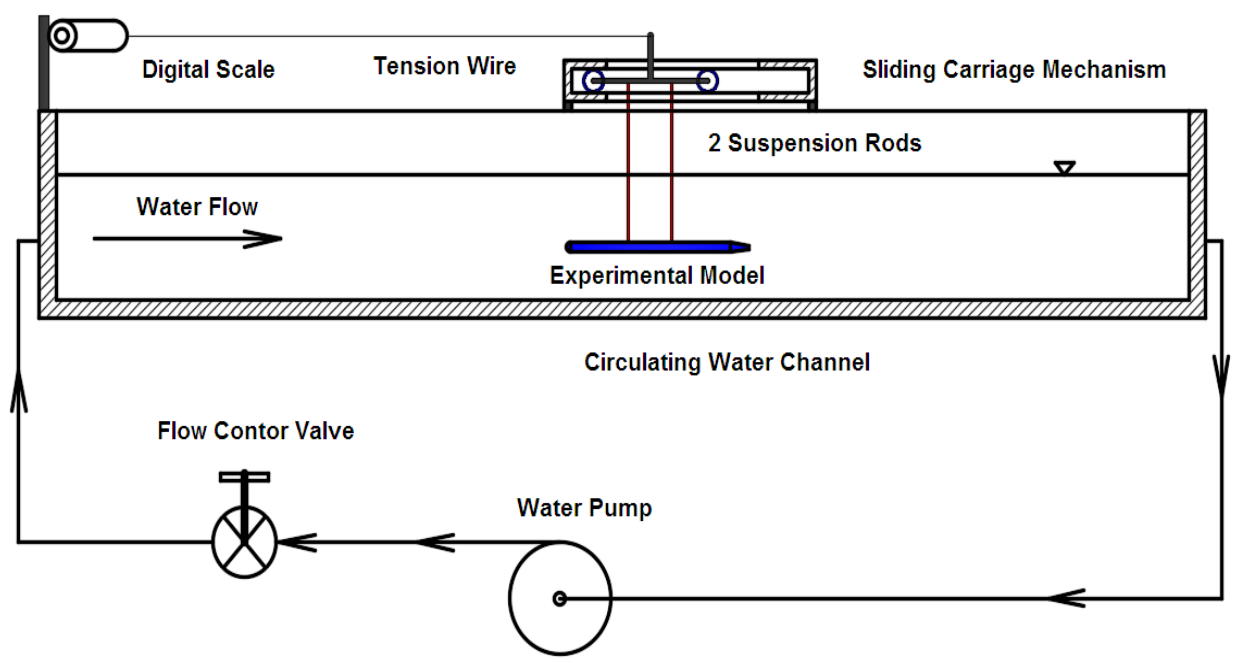

(a) Schematic diagram of the test rig.

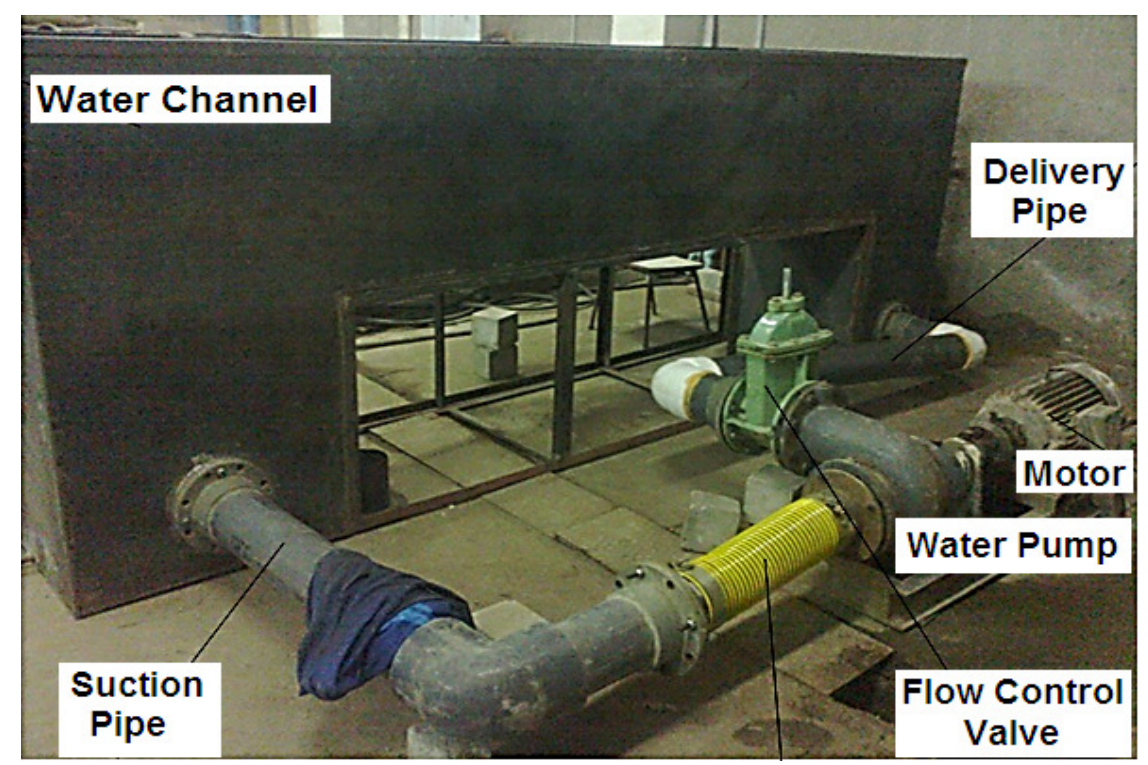

(b) Photograph of the test rig.

Fig. 2. Layout of the experimental test rig. 


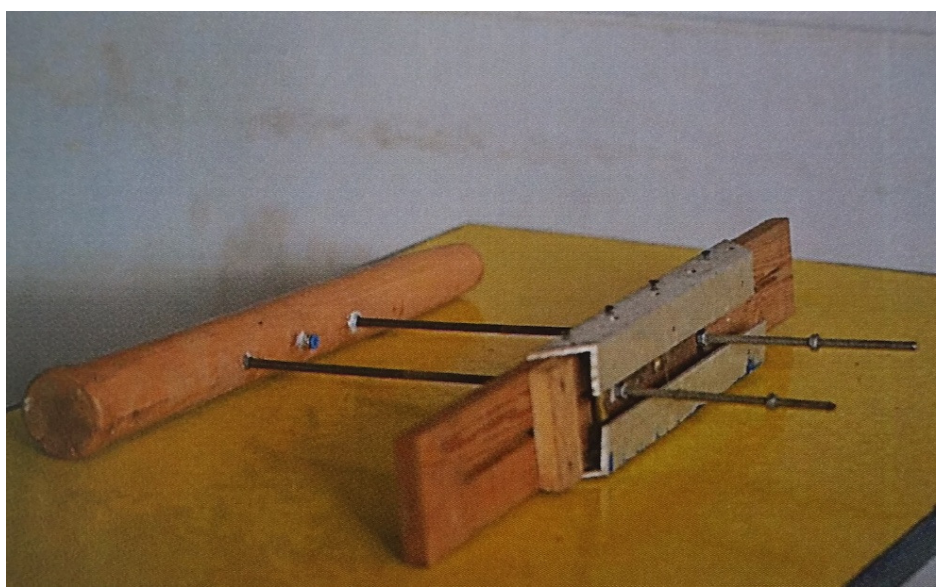

Fig. 3. A photograph of the sliding carriage mechanism, suspension rods and experimental model (10).

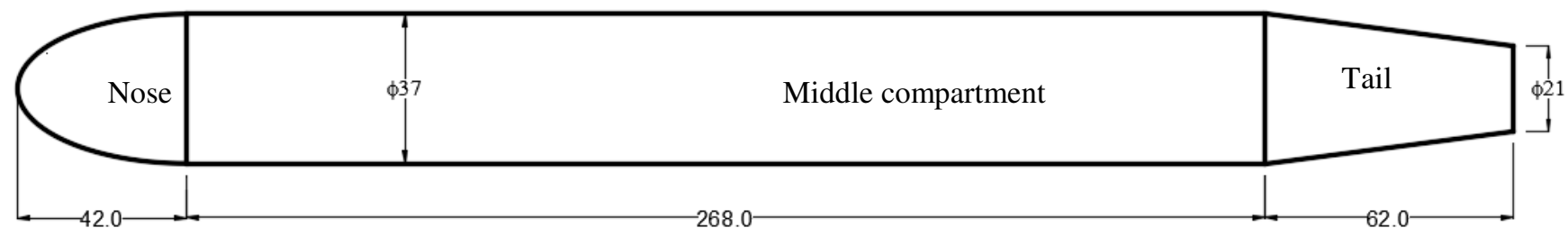

(a) The geometric dimensions of a complete modelof an under water vehicle.

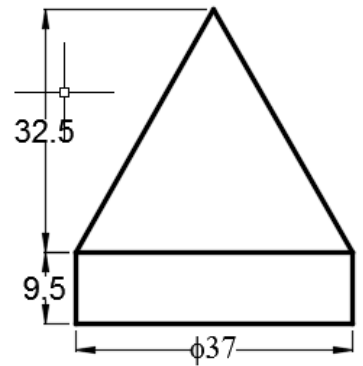

Conical nose profile Model (1)

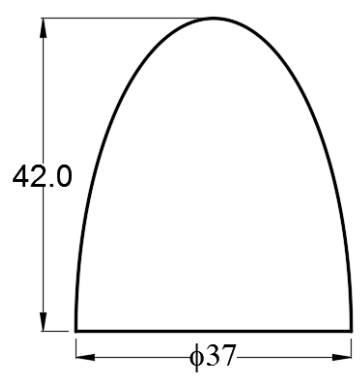

Elliptical nose profile Model (5)

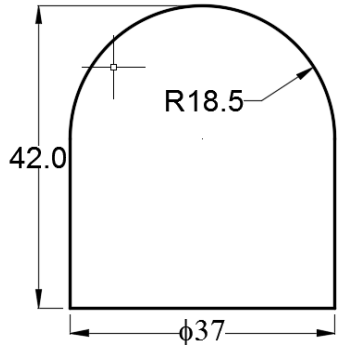

Hemispherical nose profile Model (2)

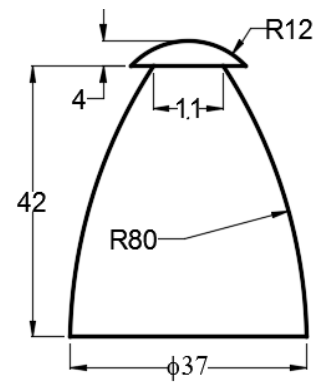

Super-cavitation nose profile (1), Model (6)

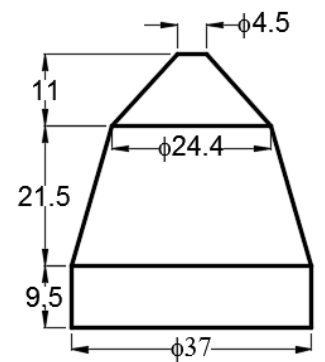

Stepped Conical nose profile Model (3)

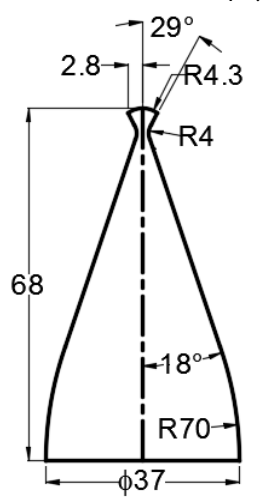

Super-cavitation nose profile (2), Model (7)

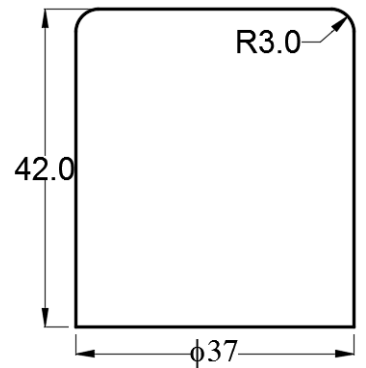

Stubbed nose profile Model (4)

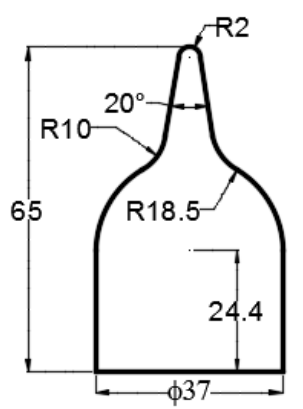

Dolphin nose profile Model (8)

(b) Eight different nose profiles considered in the investigation

Fig. 4. The geometric dimensions of the investigatedmodels. 


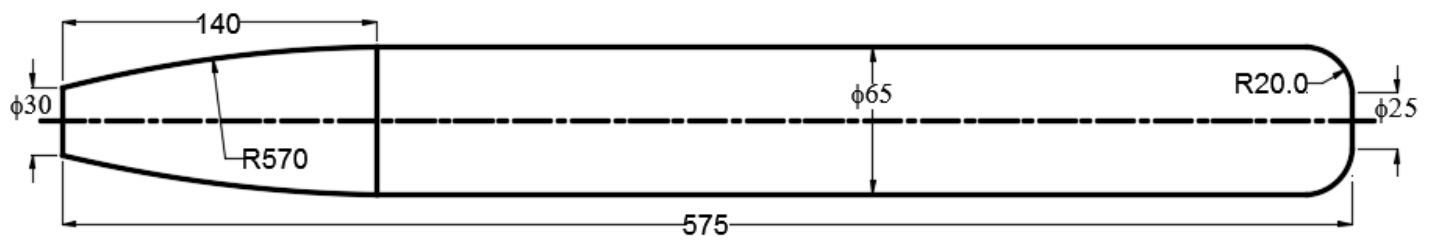

Fig. 5. The geometric dimensions of theexperimental model of Ref. [9], Model (9).

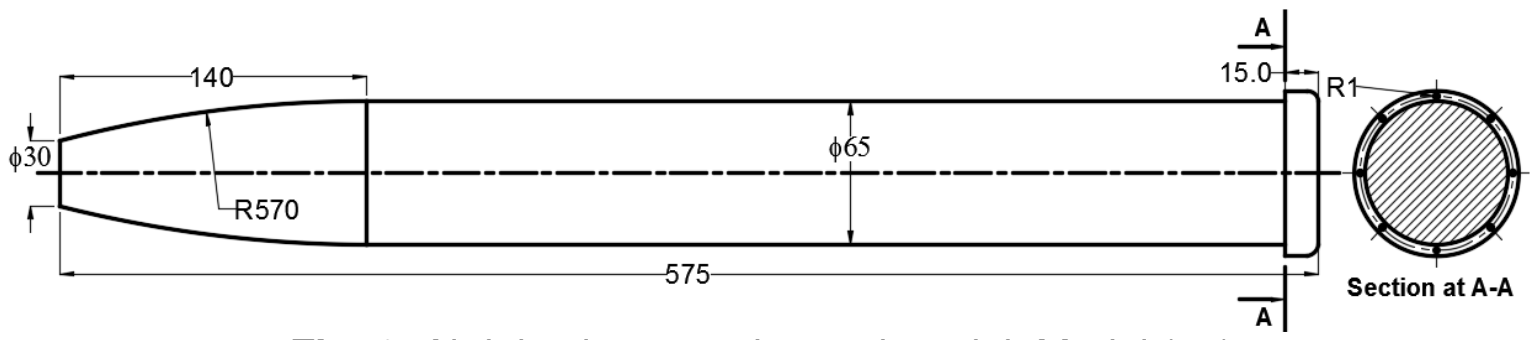

Fig. 6. Air injection experimental model, Model (10).

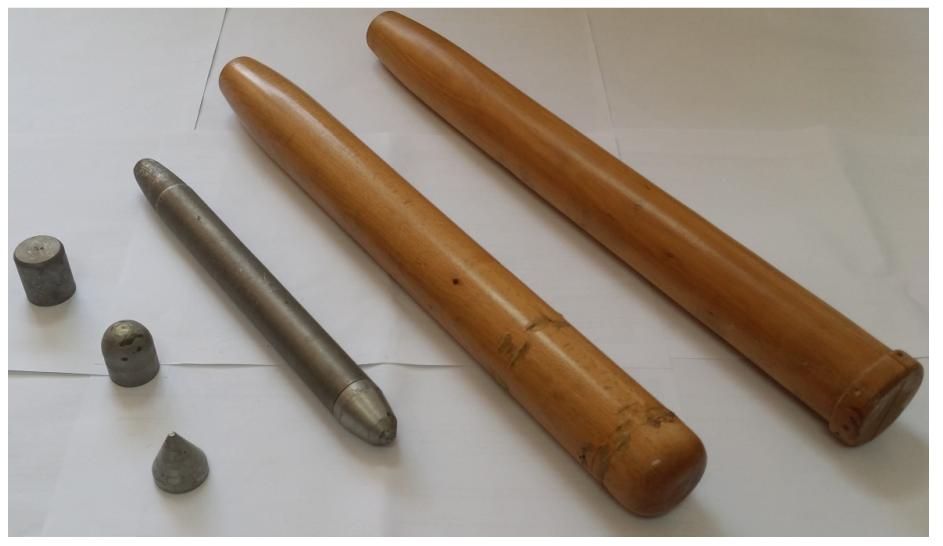

Fig. 7. A photograph of the six experimental models.

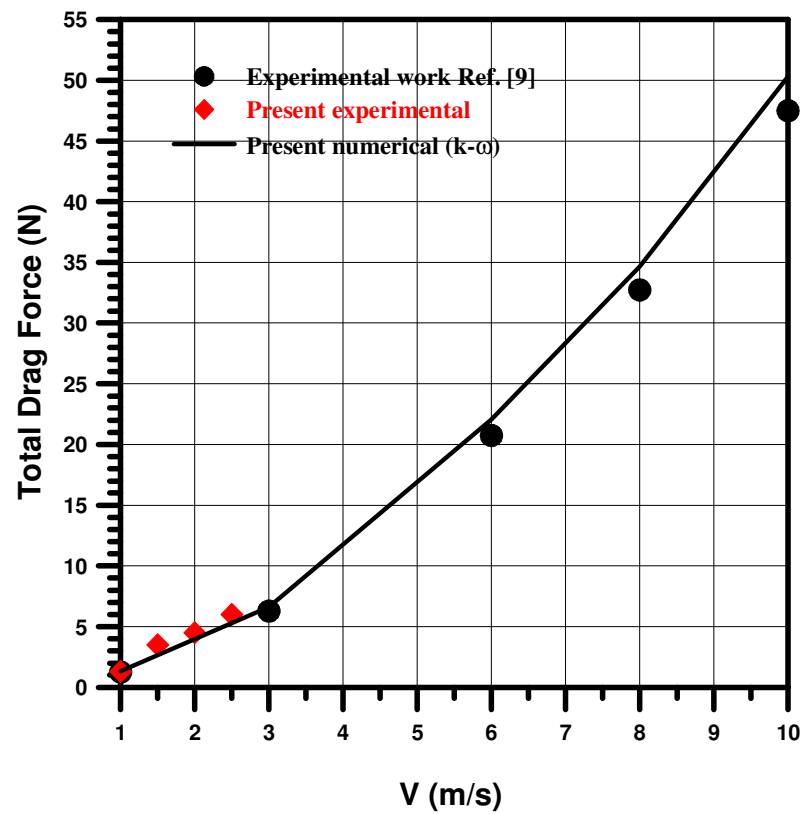

Fig. 8. Validation of present numerical and experimental total drag forces of model

(9) against experimental data provided by Ref. [9]. 


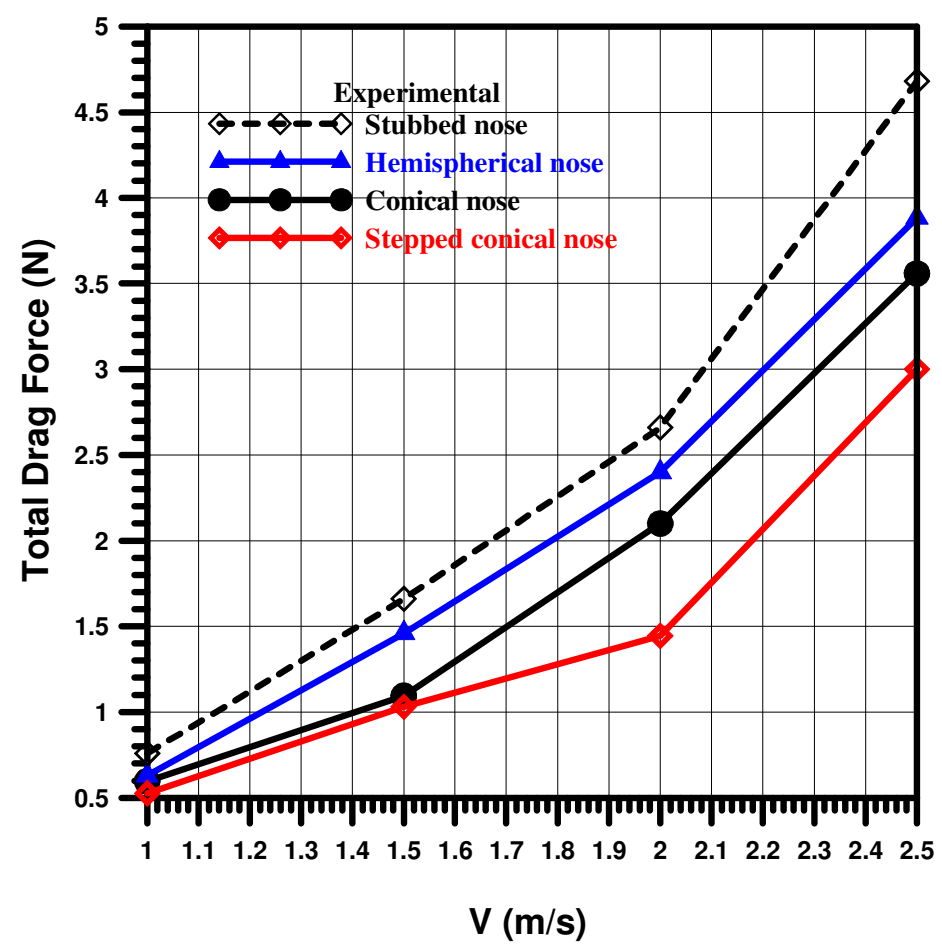

Fig. 9. Comparison of measured total drag force of models (1 to 4 ).

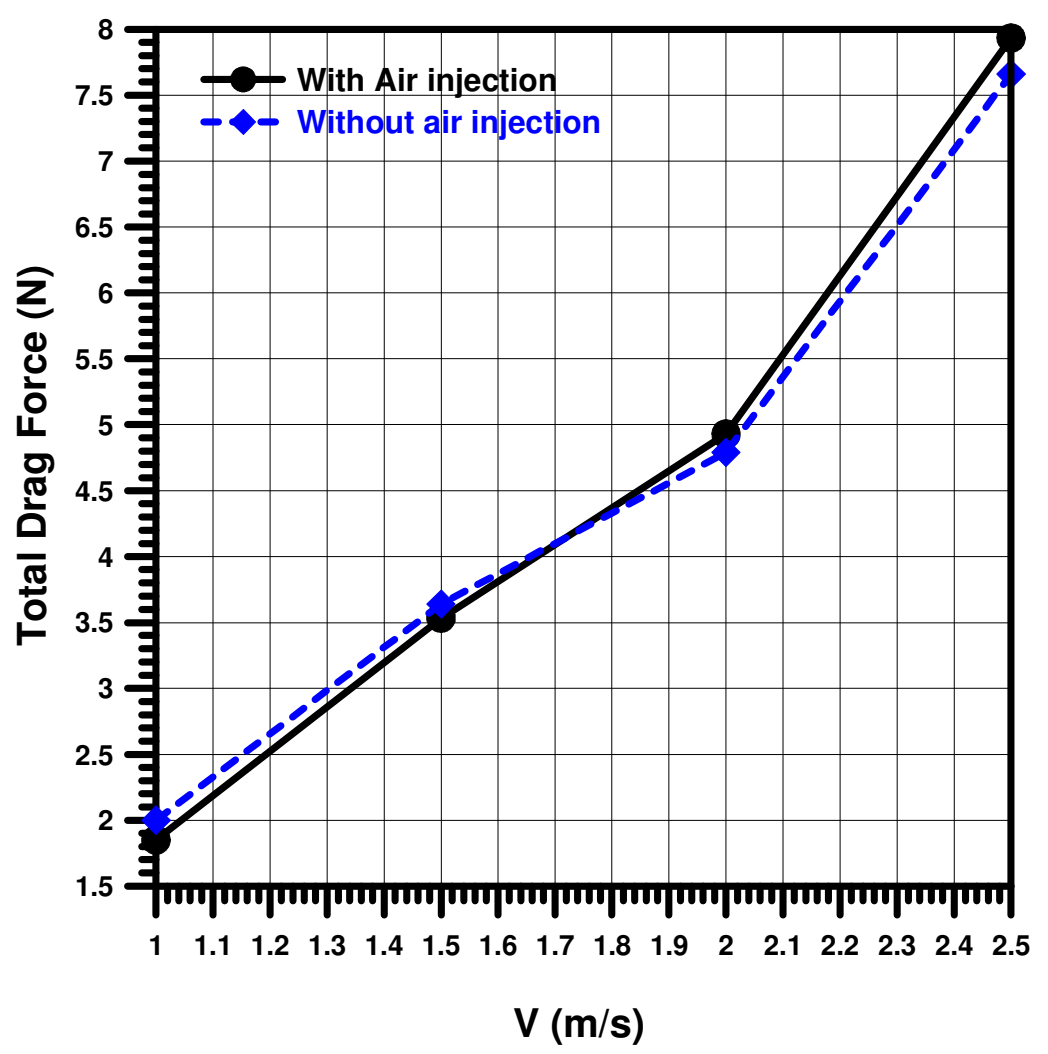

Fig. 10. Comparison of measured total drag force of model (10) with and without air bubbles injection. 


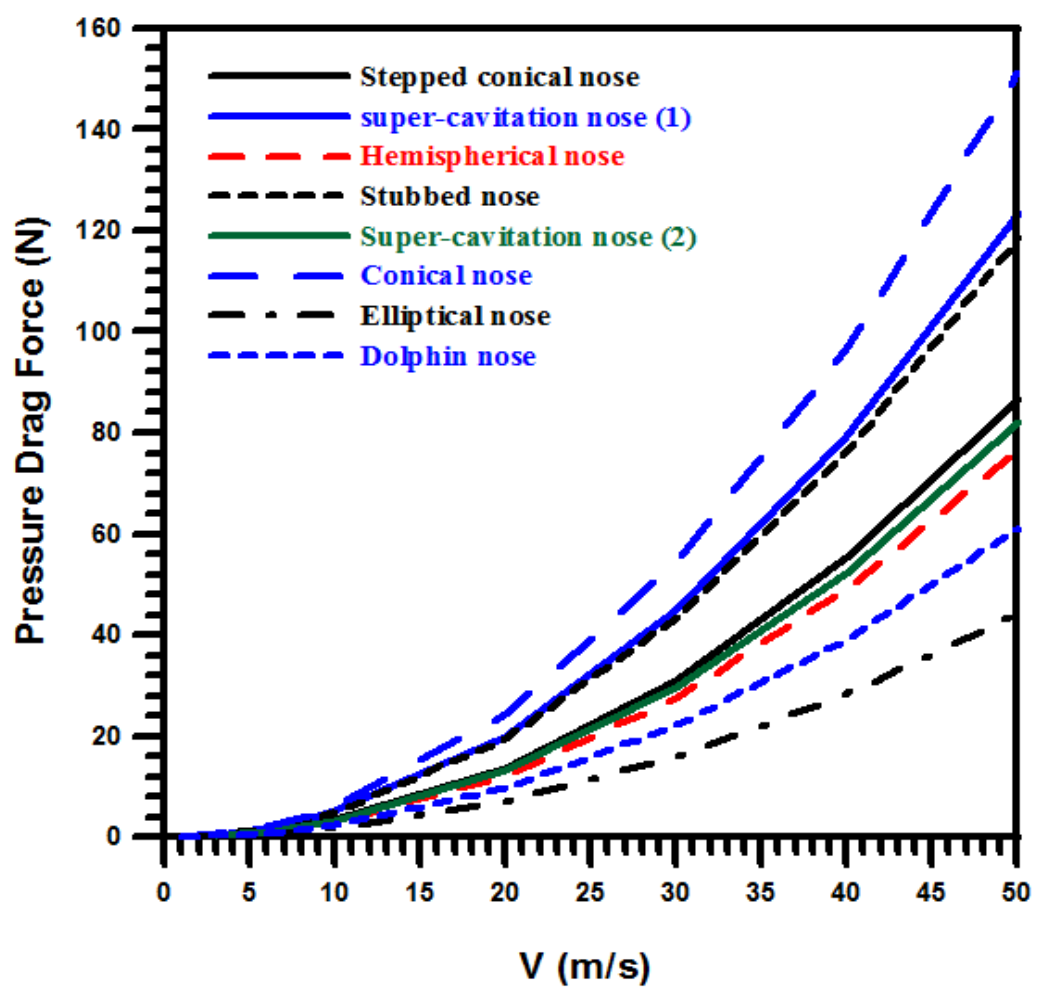

Fig. 11. Predicted pressure drag force of eight different nose profiles.

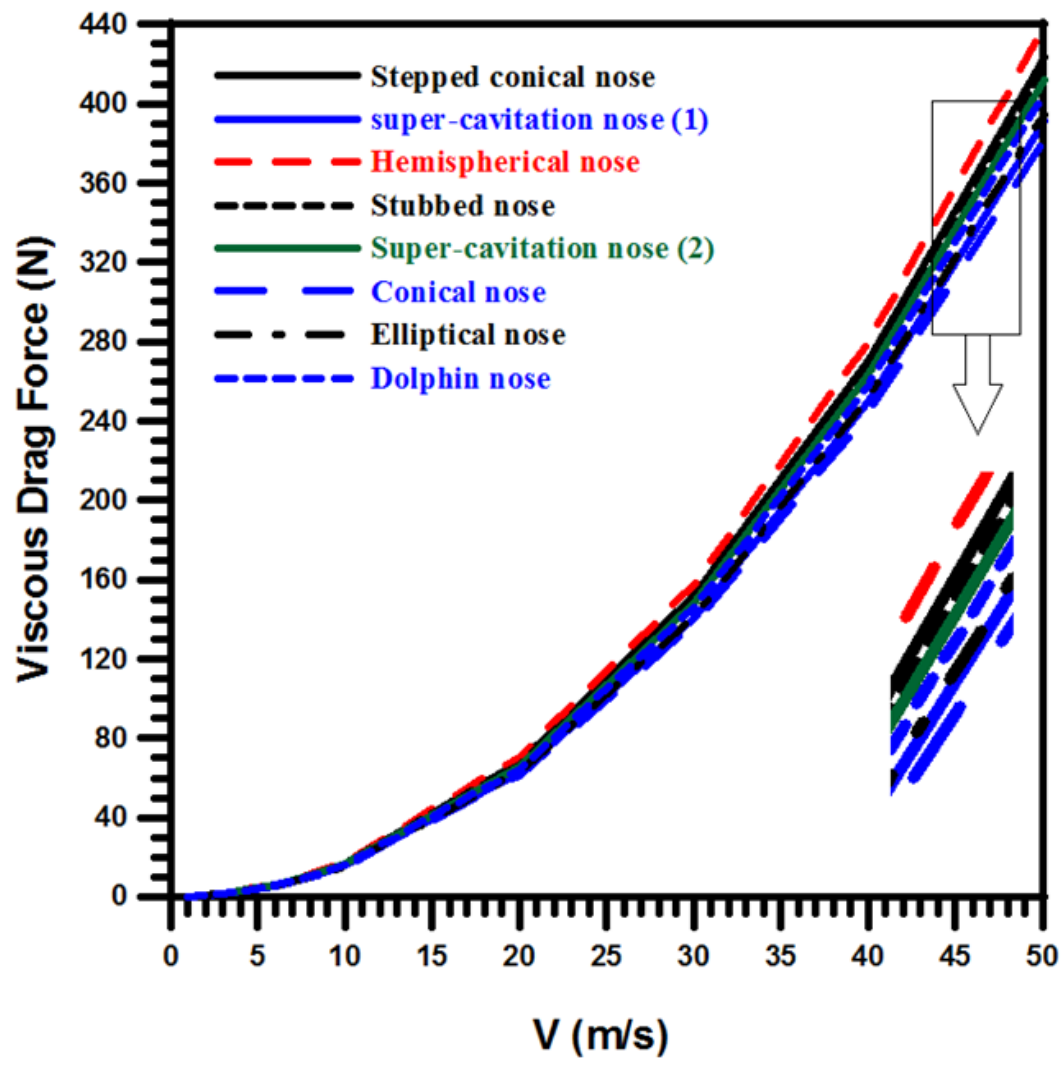

Fig. 12. Predicted viscous drag force of eight different nose profiles. 


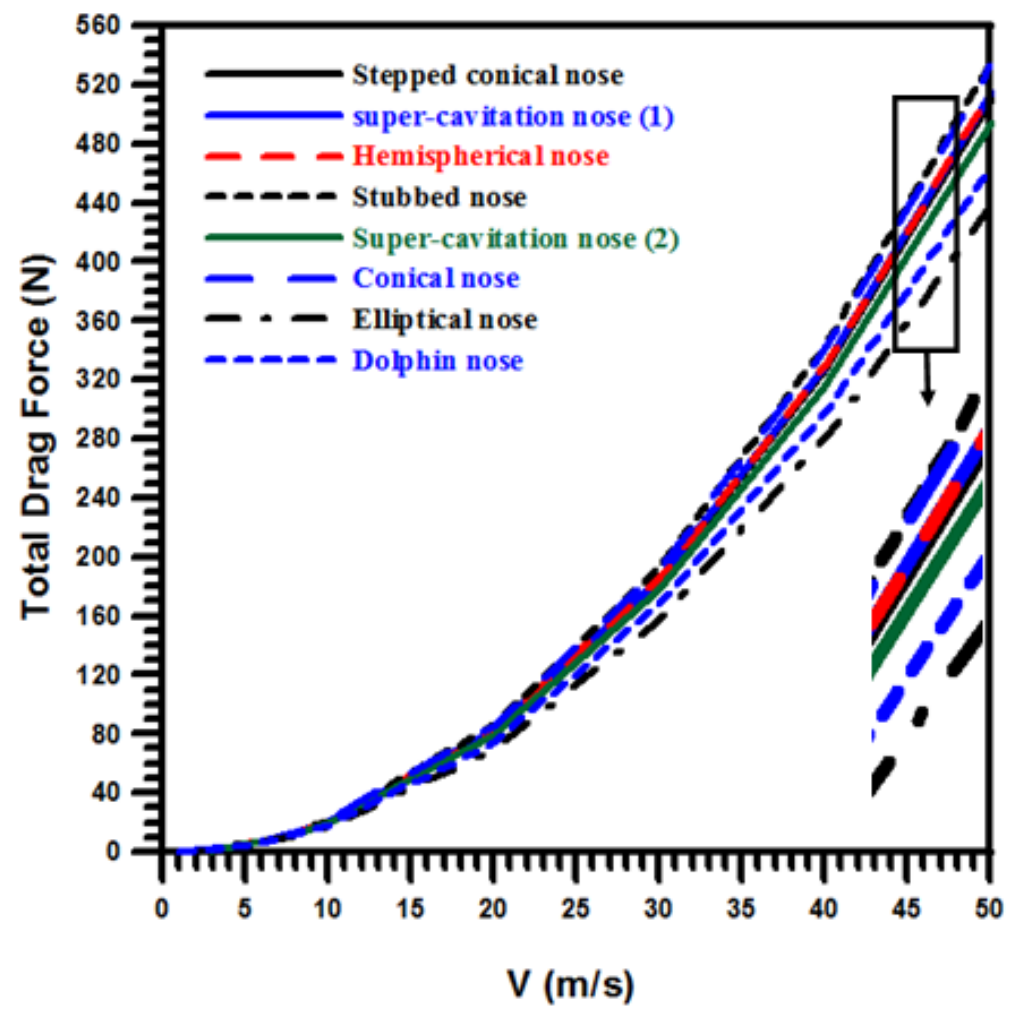

Fig. 13. Predicted total drag force of eight different nose profiles.

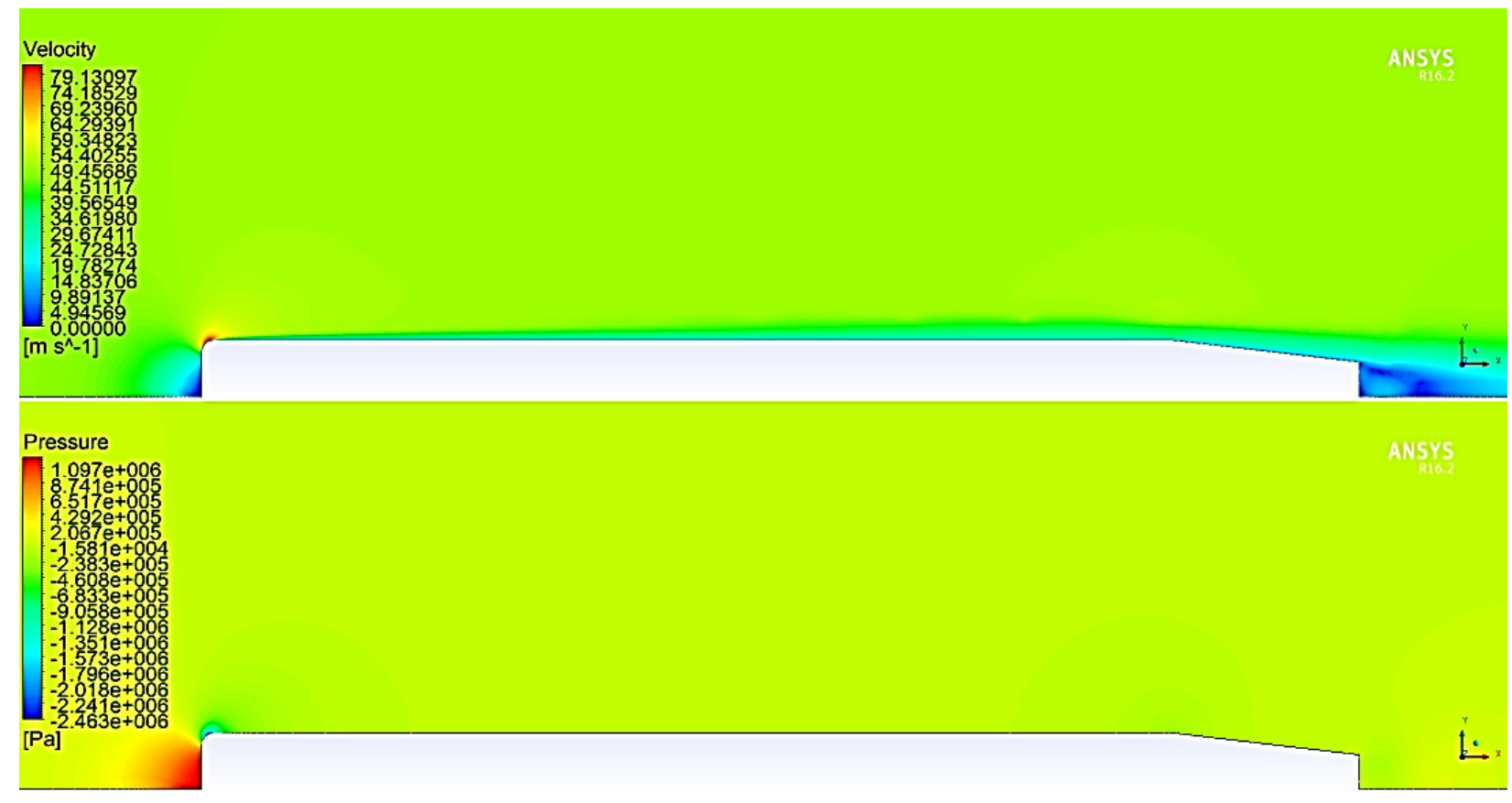

Fig. 14. Contours of velocity and pressure of stubbed nose profile, model (4), at velocity of $50 \mathrm{~m} / \mathrm{s}$. 


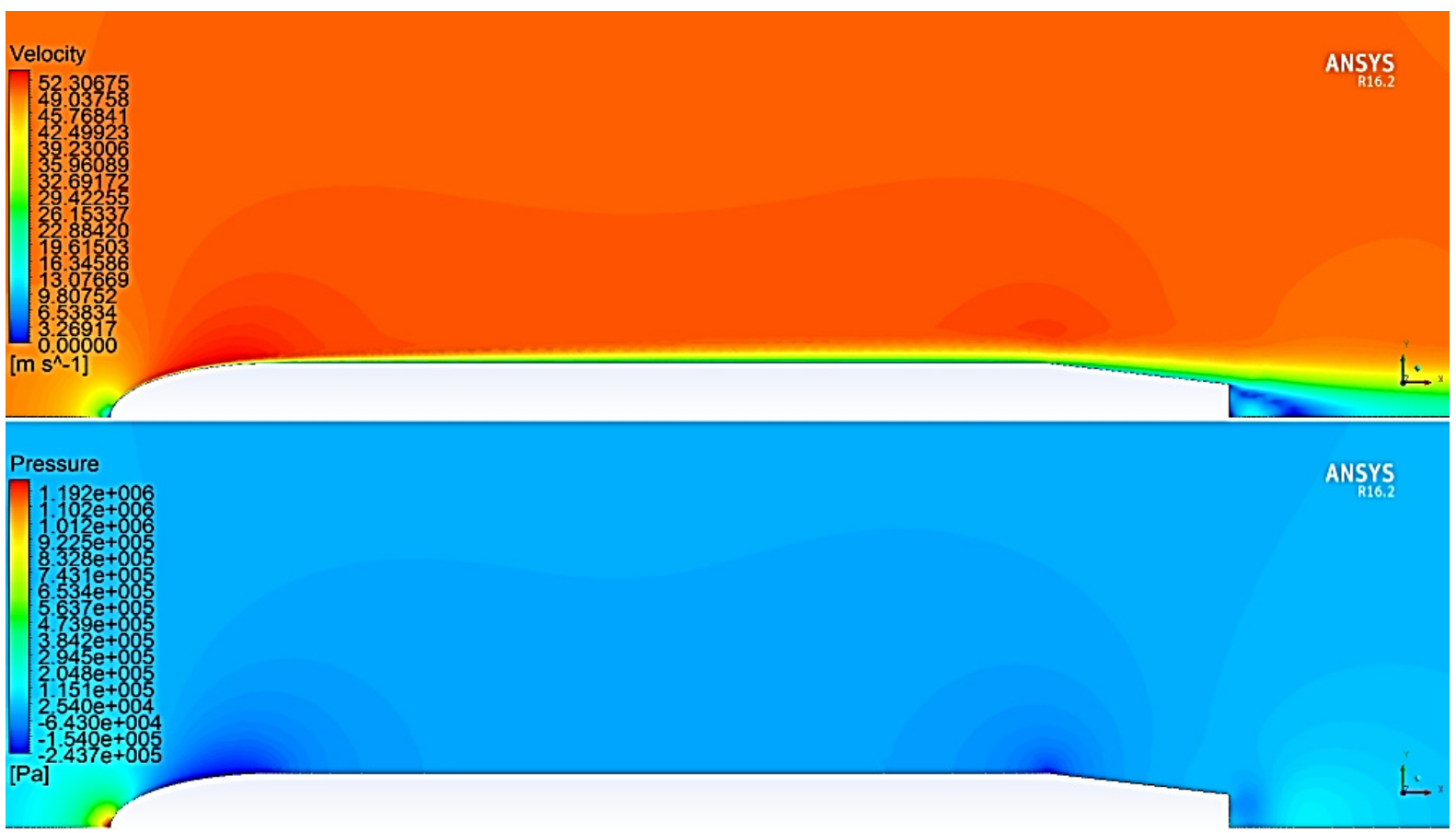

Fig. 15. Contours of velocity and pressure of elliptical nose profile, model (5), at velocity of $50 \mathrm{~m} / \mathrm{s}$. 